\title{
Mechanisms and Capabilities for Human Robot Collaboration
}

\author{
Claus Lenz ${ }^{1}$, Alois Knoll ${ }^{1}$
}

\begin{abstract}
This paper deals with the concept of a collaborative human-robot workspace in production environments recapitulating and complementing the work of the author presented in [1]. Different aspects regarding collaboration are discussed and applied in an exemplary scenario. Modalities including visualizations and audio are used to inform the human worker about next assembly steps and the current status of the system. The robot supplies the human worker with needed parts in an adaptive manner to prevent errors and to increase ergonomic benefits. Further, the human worker can intuitively interact and adjust the robot using projected menus on the worktable and by force-guidance of the robot. All these functions are brought together in an overall architecture.
\end{abstract}

\section{INTRODUCTION}

Robots have proven their success since their introduction in the industry in the late 60ties - mainly because robots are reconfigurable and therefore applicable for a variety of tasks. Although robots are flexible and multi-purpose machines, their flexibility heavily reduces due to their static task programming. If the task, the product or the environment changes, their movements often need to be re-programmed from scratch. The costs of task-adequate robots and the effort to set-up, program, and integrate them into existing production lines amortize only with a large number of manufactured products, because the costs of the integration of a robot are approximately ten times the price of the robot itself [2].

Nowadays, as manufacturing industry is faced with increasing product variants while production cycles decrease, conventional strategies to optimize production steps are saturating. Future competitiveness of manufacturers will be highly related to their ability and flexibility to adapt to these essential market requirements [3]. This flexibility is at the moment hardly reachable with fully automated production processes. Especially, if small lot sizes of units or prototypes with a high variety and high task complexity are needed, current automation strategies are not cost efficient [4].

The collaboration between human and robot has been announced as a promising approach to solve these challenges, because it teams the strength and the efficiency of robots with the high degree of dexterity and the cognitive capabilities of humans into a flexible overall system. As consequence of current flexible automation techniques including flexible manufacturing systems (FMS) and reconfigurable manufacturing systems (RMS) [5], a recent trend in robotics focuses on new generations of robots with the capability to directly assist humans. This bridges the gap between fully automated

\footnotetext{
${ }^{1}$ C. Lenz and A. Knoll are with the Department of Informatics, Robotics and Embedded Systems, Technische Universität München
}

systems and fully manual workstations [6]. Highly related is that a significant amount of research has been done in the area of physical human-robot interaction (pHRI) [7], [8] that can also be applied to collaborative tasks between human and robot. Additionally, the introduction of cognitive capabilities [9] for assistive robotic systems, new robot control schemes, and advances in artificial perception, to name only a few, are enabling factors to bring human and robot further together in a shared workspace. Especially, technical systems need to improve their performance with respect to unforeseen events, flexibility in their use, and field of application through cognitive capabilities [3]. This creates more flexible and (partly) autonomous machines that are able to directly cooperate and support the human coworker [10], [11]. Further, keeping the human in the loop of production processes for highly flexible assembly advances the skills of the overall system due to cognitive and sensomotoric advantages of the human. Hence, the human is part of production processes when he is needed and can concentrate on other tasks to improve the overall system performance [12].

The support of humans by robotic systems can then lead on the one hand to more ergonomic work places and on the other hand to more time-efficient production processes. Additionally, the amount of fixed production costs in relation to variable costs can be reduced [13]. The advantage of the potentials for humans and robots to work together as a team is only in early stages and needs a safe, robust and efficient realization [14]. Once this is reached, the subsequent flexibility and adaptability of human and robot collaborating as a team allows production scenarios in permanently changing environments as well as the manufacturing of highly customized products in factories of the future.

This paper recapitulates and complements the work of the author published in [1].

\section{COllaborative (INDUSTRIAL) ROBOTIC SYSTEMS}

Robotic systems that assist human workers in production processes as well as in production environments are an active research field with a variety of applications. The following overview gives an impression about systems that have been introduced in the past to tackle human-robot collaboration in the production.

A robotic system consisting of multiple impedancecontrolled robots is introduced by Kosuge in [15]. With this system, a joint object manipulation of human(s) and (multiple) robot(s) in a dynamic way is possible. The control scheme assumed that the interaction takes always place through an object. A force sensor attached to the wrist of 
each robot measured the external forces on the robots through the object. The human commanded the movement of the robots by applying forces to the object. This approach was later extended to the mobile robotic assistant MR Helper as presented in [16].

Khatib presented in [17] several strategies to support workers in physical tasks for compliant motion and cooperative manipulation. In addition to the controlling of multiple arms corresponding to the applied forces, multiple holonomic mobile platforms were coordinated to have a fully flexible mobile assistant.

For situations that involve large interaction forces as it is for example present in the automobile production, Cobots have been introduced by Colgate in [18]. These specialized mechanical devices provide guidance to human operator's motion. The cobots act passively with virtual fixtures and virtual walls to support and guide the human collaborator without the intention to act autonomously.

PowerMate - introduced by Schraft in [12]—is another example of a system designed to give the human worker a robotic assistant for handling and assembly tasks. The system follows current safety norms and works with normal velocity, if no human is present. In presence of a human, the velocity is limited and with the confirmation of the human, the robot can be guided to place heavy good using force/torque sensing. With this mode, it is possible to pull the robot on its gripper to a desired position.

A rather application oriented approach to ease and speed up the programming of industrial robots is presented by Pires in [19]. The approach is object-oriented and based on a client-server architecture. It is claimed that the underlying concept is general enough to be applied to organize and program overall flexible manufacturing cells.

Flexible, adaptive, and cognitive robots are especially needed, if small lot sizes of units or prototypes with a high variety and high task complexity are required. That means, that future industrial robotic assistants should be flexible and safe on the one side and clever helpers in manufacturing environments on the other side. Hägele describes this in [14] as the evolution from robots to robot assistants. To show the concept presented in [14], the mobile robot assistant rob@work has been developed by Helms and Hägele [20], [21] as direct interacting and flexible device for assistance. The collection of functionality includes automatic path planning, obstacle avoidance, precise positioning, and several safety concepts of the robotic arm motions. Further, the ease of use to instruct tasks was demonstrated.

A cooperative assembly cell system using a four-axis scara robot was presented by Thiemermann in [22], [23]. The system enhanced standard tasks of the assembly robot with new functionality to help the human worker with the workpiece positioning or with other parts and tools. A camerabased system is used to adapt the working velocity of the robot according to the distance between human and robot.

A mobile assistive robot for flexible and interactive manufacturing is presented by Stopp in [24]. Due to safety aspects, the human instructor teaches interactively the robot an order-picking from outside its workspace using a laser pointer and a handheld computer. This has been extended to a safety concept using dynamic sensor-based surveillance of the robot workspace and multiple safety regions with (possibly) different safety levels [25].

Iossifidis presents in [26] the stationary 7 dof robotic assistant $\operatorname{CoR} A$, that is able to cooperatively solve an assembly task using diverse inter-connected components including speech, object, and gesture recognition. Additionally, the robot has been prepared with an artificial skin to allow a touching and positioning of the robot by the human.

Gecks presents in [27] SIMERO, a stationary industrial robot system. Several stationary cameras that detect obstacles to dynamically adapt motions accordingly supervise the workspace of the robot. This approach has later been enhanced by the use of multiple depth cameras [28] to perform a three-dimensional collision avoidance for unknown objects. One master computer evaluates the synchronously acquired data of slave computers and employs a geometrical model to revise the data points and to adjust the robot velocity according to distance of human and robot. In this way collisions with obstacles or the human can be damped or even prevented.

A pro-active collaboration between human and robot based on the recognition of the intentions of the human is described by Schrempf in [29]. As the recognition of intentions is quite uncertain, the robot resolves this uncertainty by pro-active execution to minimize the overall costs. For the system, Dynamic Bayesian Networks (DBNs) were used.

The approach by Rickert presented in [30] describes a scenario in which a human and the JAST robot-a robot with two arms in a human-like arrangement-build together a wooden model of an aircraft using a distributed architecture divided into the high-level components input, interpretation, representation, reasoning, and output with several functional modules.

Another example is the DLR lightweight arm presented in [31], [32] that was transferred to $\mathrm{KUKA}^{1}$ and is now commercially available. This lightweight robot is especially designed for interaction with unknown environments and with humans [33]. The integrated compliance, virtual fixtures, high interpolation rate, and many more make the robot very promising to work alongside with humans. Haddadin uses this arm to build a sensor-based robotic co-worker for a safe and close cooperation and presents strategies for safe interaction with the human [34].

Chuang presents in [10] a study on human robot collaboration design for robot assisted cellular manufacturing and identifies that collaboration planning, collaboration safety, mental workload management, and a good manmachine interface as four main concepts that need to be met to enable human robot collaboration. Further the authors present in [35] experiment evaluations of different supportive information formats and show, that it is important to display information near relevant object and in the visual attention

\footnotetext{
${ }^{1}$ http: //www.kuka.com
} 
field of the human. Additionally, the combination of information sources such as text and images is important.

\section{PSYCHOLOGICAL ASPECTS}

To successfully integrate collaborative assembly systems in today's processes, high demands regarding safety, efficiency, ergonomics, flexibility, programmability, and adaptability need to be met. As humans being experts in physical interaction and collaboration, the investigation of high-level joint-action strategies between humans is important to find strategies for robotic systems [36]. Further, the research on especially humanoid robotics can on the reverse side also contribute to understand how humans behave [37]. Psychological research on cognitive processes of joint-action among humans [38] lists task sharing, joint attention, action observation and action coordination as important mechanisms that influence the efficiency:

- joint attention to steer ones concentration and to share representations about events and objects

- task sharing to be able to predict the next steps based on the expected behavior of the opponent before an action can be observed

- action observation to predict the next goal based on the current behavior of the opponent

- and action coordination to adjust own actions in space and time to the behavior of the opponent

That means, that in collaborating human-human teams, an efficient coordination requires participants that plan and execute their actions in relation to what they expect from the opponents based on observations [39]. Action of the team members are observed and evaluated to coordinate own actions appropriately in space and time. Hence, humans negotiate unconsciously various parameters to optimize the co-operation during the collaboration [40]. During repetitions of the same action, the coordination becomes smoother and more accurate and leads to a maximum in comfort and efficiency.

Additionally, enabling factors are the abilities of the system to act autonomously in the environment according to sensor and context information. The communication and the explanations from the assistive system should also follow psychological aspects to decrease the distraction and the cognitive load of the human [41], [42].

Further, technical systems should make use of information provided by multiple sensors, (multiple) actuators, that are embedded in and aware of the real world to perceive, reason, learn and plan in a cognitive way. Along with reflectiveness about their own capabilities and limitations, cognitive technical systems know what they are doing, how things can be done, and about the human collaboration partner. This leverages higher flexibility, adaptivity, interaction and collaboration capabilities of the systems [43].

Another important issue constitutes, that collaboration of partners can be defined as being based on achieving a common goal together with commitments of every participating partner. This differs significantly from short-term interaction, where partners have no shared (long-term) goal [44]. Although, it is hard to distinguish these two terms in many cases at first glance, the difference becomes clear if errors occur: partners that act jointly and collaborative with the same global goal in mind can support each other and assist, because both know what needs to be done [45].

Therefore, to manage a predefined goal-e.g. the joint assembly of a product - the robot-system needs to know about the task in the production process as well as the human worker (task sharing). If the representation of the subtasks is as generic as possible, the role allocation can dynamically change even during the execution. A common representation of human and robot capabilities is an important issue in order to assign tasks according to specific skills [46]. With the knowledge about a shared plan, the system is able to predict possible next action steps and prepare these steps pro-actively.

For cooperation between robot and human, it is important that both partners coincide on the same objects and topics and create a perceptual common ground with a shared representation [38]. Considering joint attention, the system needs to have the ability to control the focus-of-attention of the human to direct to relevant objects or has to be able to estimate the human's focus-of-attention directly. This can also include pointing gestures as integrated in [30] or the head orientation of the human worker.

The transfer of these basic mechanisms of joint-action to a robotic assistive system can improve the collaboration of human and robot. This also includes the unconscious adaption of own actions using anticipatory knowledge about the actions of the team member and the recognition of what the team partner is currently doing. The benefit of transferring anticipatory action to a human-robot context is also shown in [47], where a significant improvement of task efficiency compared to reactive behavior was possible. For an extensive evaluation of these hypothesis, we want to refer to previous publications [1], [48], [49], [50].

\section{IMPLEMENTATION}

The mechanism to enable a collaboration between human and robot described in the previous sections require certain capabilities of the system. We see perception, recognition, dynamic and adaptive motions, and communication as central capabilities for a human-robot collaborative system. Therefore, we have implemented and evaluated these capabilities as follows:

\section{A. Capability: Perception}

Knowledge about the static and dynamic situation is essential for joint workspaces of human and robot. Surveilling the joint area helps to realize a collaboration and co-existence of human and robot. Therefore, we have propose in [51] a system that redundantly monitors the workspace to perceive obstacles within the workspace including the human worker. We use multiple, distributed range sensors (i.e. Microsoft Kinect) and de-centrally pre-process the data from the sensors. These data sets are further processed and segment and 


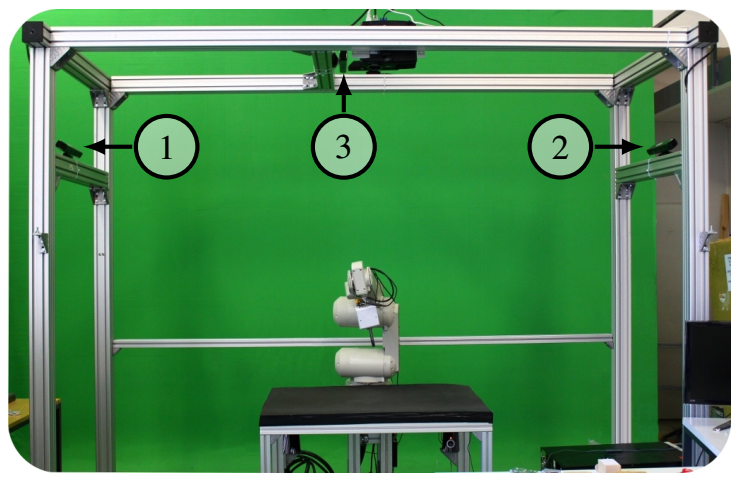

(a) The JAHIR set-up

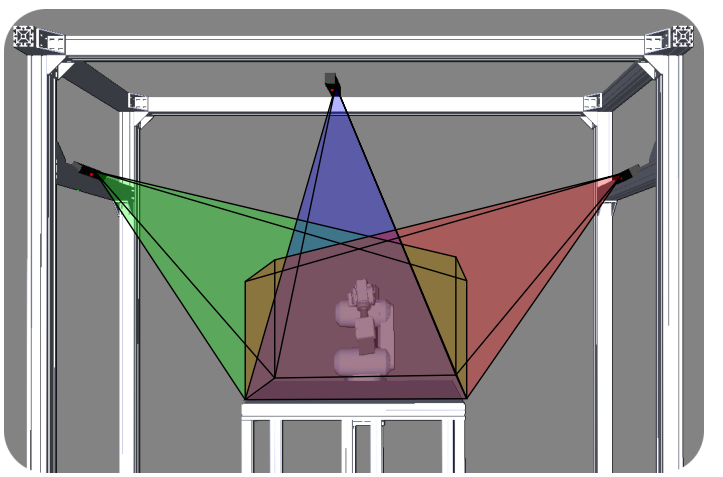

(b) Visualization of the JAHIR set-up

Fig. 1. Positions of Kinect sensors. Kinect devices 1-3 are installed on a scaffold surrounding the workbench (a). The devices are also integrated into the three-dimensional simulation of the set-up (b). The view areas of the devices that are of interest for the workspace surveillance are also highlighted in (b)

cluster unknown objects. The system finds and segments individual object point clusters in the acquired point clouds. For each detected object point cluster, a convex hull is computed which approximates the shape of the underlying object. The detected objects are then integrated into the global environment model. With the help of the integrated filtering methods, the system is able to distinguish between the static environment, the robot and other obstacles in the surroundings at high update rates. The gained geometric information can then be used in the robot controller to allow the system to react accordingly.

\section{B. Capability: Recognition}

In [52], we have presented a Hidden Markov Model (HMM) based workflow analysis of an assembly task jointly performed by a human and an assistive robotic system. In an experiment subjects had to assemble a tower by combining six cubes with several bolts for their own without the influence of a robot or any other technical device. To estimate the current action of the human, we have trained composite HMMs. After the successful evaluation on disjunct experimental data sets, the models are transferred to our assistive robotic system, where the same assembly tasks was executed. A new 3D occupancy grid approach was used to determine the hand positions of the worker. The positions were then used to compute the inputs of the analysis HMMs. The workflow of the right hand could be recognized with an accuracy of 92.26

\section{Capability: Dynamic and adaptive motions}

In [49] we have presented investigation on the influence of different hand-over timing strategies on the fluency and efficiency of such a human-robot team. Four different timing strategies were experimentally per- formed with 37 volunteers: (I) a fixed time interval between two hand overs, (II) a reactive behavior, where the robot is triggered by the human, (III) a fixed time intervals depending on the current component, and (IV) a predictive assembly duration estimation algorithm. During the experiment, the time- tocompletion of the task and the waiting times for human and robot were measured as reciprocal indication for the efficiency and respectively the fluency of the team. The evaluation of the experiment indicates that the efficiency of the human-robot team is significantly increased using a predictive timing strategy, because it enables the robot to provide the subsequently needed component just-in-time. The decrease in waiting times for both human and robot approves increasing fluency of the collaboration. Additionally, the time prediction provides the opportunity to perform additional tasks with the robot, while the worker is occupied. Hence, nearly a full use of both partners' capacity in a assembly scenario can be achieved.

\section{Capability: Communication}

We have equipped our system with a variety of modalities to communicate with the human worker. On the output side we have

- a text-to-speech module to instruct the human and to give feedback

- an on-table projection to adaptively instruct the human and give feedback about the system status

- the adaptive robot itself, that is adaptively acting (see above).

On the other hand, the human has different possibilities to interact with the system. We have implemented

- a force control to adjust the robot

- a module that surveils the workspace and tracks the human (full body)

- virtual projected buttons and menus to (high-level) control the robot and the assembly process

\section{CONCLUSION}

In this paper, we have recapitulated on mechanism of human and robot collaboration and their instantiation through different capabilities to keep the human in the loop. The capabilities have been partly evaluated in previous work of the author. With this paper, the results are brought together in a broader context of joint human-robot action. An instance of the evaluation platform is depicted in the images of figure 2. A video showing the integration into the system in a joint collaboration can be found here: http://www - youtube. $\mathrm{com} /$ watch? $\mathrm{v}=\mathrm{GpXkEd6y1LE}$. 


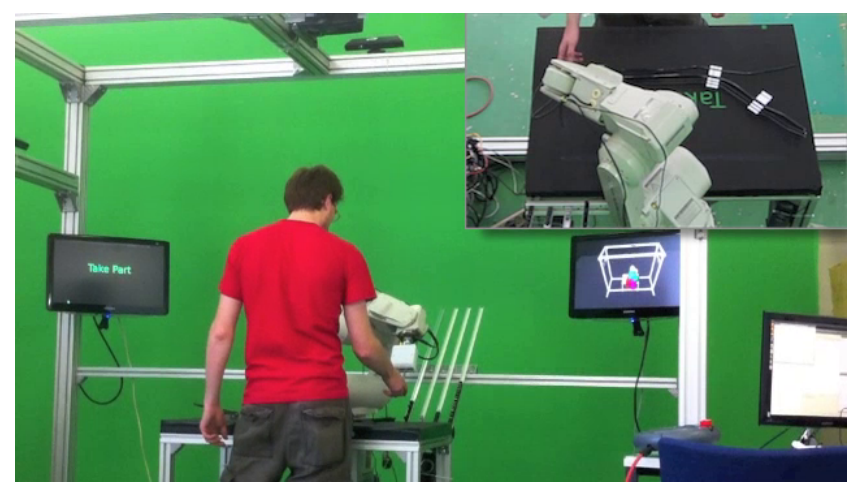

(a)

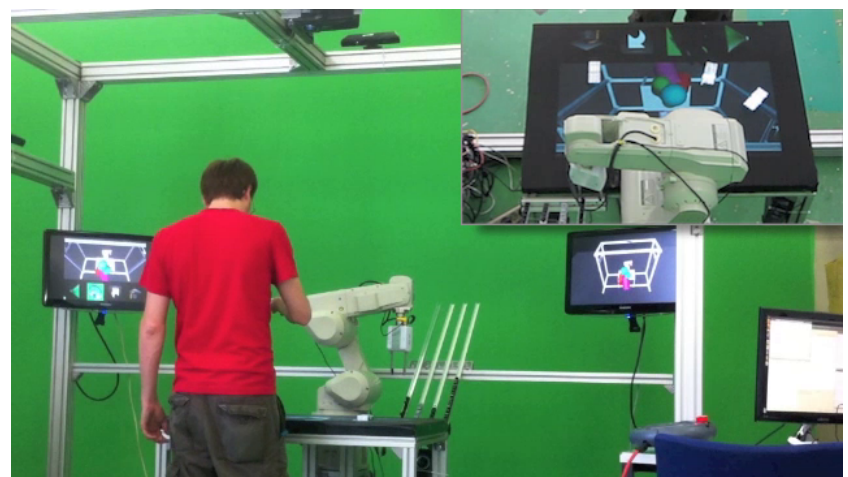

(b)

Fig. 2. Human Robot Collaboration Plattform. The human can interact with a collaborative robot in a natural manner. The workspace is augmented with multiple screens showing the system status and instructions to the human. (a) The human receives a part from the robot. (b) The human can interact with the system using virtual buttons projected on the work desk.

\section{REFERENCES}

[1] C. Lenz, "Context-aware human-robot collaboration as a basis for future cognitive factories," Dissertation, Technische Universität München, München, 2011.

[2] S. Bartscher, "Mensch-Roboter-Kooperation in der Montage," in Münchner Kolloquium-Fachformum Automation und Montagetechnik, H. Hoffmann, G. Reinhart, and M. F. Zäh, Eds., 2010, pp. 33-40.

[3] A. Bannat, T. Bautze, M. Beetz, J. Blume, K. Diepold, C. Ertelt, F. Geiger, T. Gmeiner, T. Gyger, A. Knoll, C. Lau, C. Lenz, M. Ostgathe, G. Reinhart, W. Rösel, T. Rühr, A. Schuboe, K. Shea, I. S. genannt Wersborg, S. Stork, W. Tekouo, F. Wallhoff, M. Wiesbeck, and M. F. Zäh, "Artificial cognition in production systems," IEEE Transactions on Automation Science and Engineering, vol. PP, no. 99, pp. 1-27, July 2010.

[4] M. Zäh, M. Beetz, K. Shea, G. Reinhart, K. Bender, C. Lau, M. Ostgathe, W. Vogl, M. Wiesbeck, M. Engelhard, C. Ertelt, T. Rühr, and M. Friedrich, Changeable and Reconfigurable Manufacturing Systems. Springer, 2009, ch. The Cognitive Factory, pp. 355-371.

[5] Y. Koren, U. Heisel, F. Jovane, T. Moriwaki, G. Pritschow, G. Ulsoy, and H. V. Brussel, "Reconfigurable manufacturing systems," CIRP Annals - Manufacturing Technology, vol. 48, no. 2, pp. 527 540, 1999. [Online]. Available: http://www.sciencedirect.com/science/ article/B8CXH-4P4GHGD-4/2/e4a63be5f1dcfd96c96f802f012d33fe

[6] J. Krüger, T. Lien, and A. Verl, "Cooperation of human and machines in assembly lines," CIRP Annals - Manufacturing Technology, vol. 59, 2009.

[7] R. Alami, A. Albu-Schaeffer, A. Bicchi, R. Bischoff, R. Chatila, A. D. Luca, A. D. Santis, G. Giralt, J. Guiochet, G. Hirzinger, F. Ingrand, V. Lippiello, R. Mattone, D. Powell, S. Sen, B. Siciliano, G. Tonietti, and L. Villani, "Safe and dependable physical human-robot interaction in anthropic domains: State of the art and challenges," in Proceedings IROS'06 Workshop on pHRI - Physical Human-Robot Interaction in Anthropic Domains. IEEE, 2006.

[8] V. Duchaine and C. Gosselin, "Safe, stable and intuitive control for physical human-robot interaction," in Proceedings of the IEEE International Conference on Robotics and Automation, Kobe, Japan, May 2009, pp. 3383-3388.

[9] E. Hauck, A. Gramatke, and K. Henning, "A software architecture for cognitive technical systems suitable for an assembly task in a production environment," in Automation Control - Theory and Practice, 2009.

[10] J. T. C. Tan, F. Duan, Y. Zhang, K. W. abd Ryu Kato, and T. Arai, "Human-robot collaboration in cellular manufacturing: Design and development," in Proceedings of the IEEE/RSJ International Conference on Intelligent Robots and Systems, St. Louis, USA, oct 2009, pp. 2934.

[11] T. Ogure, Y. Nakabo, S. Jeong, and Y. Yamada, "Risk management simulator for low-powered human-collaborative industrial robots," in Proceedings of the IEEE/RSJ International Conference on Intelligent Robots and Systems, St. Louis, USA, oct 2009, pp. 49-54.
[12] R. D. Schraft, C. Meyer, C. Parlitz, and E. Helms, "Powermate-a safe and intuitive robot assistant for handling and assembly tasks," in Proceedings of the 2005 IEEE International Conference on Robotics and Automation, 2005, pp. 4074-4079.

[13] J. Krüger, R. Bernhardt, D. Surdilovic, and G. Spur, "Intelligent assist systems for flexible assembly," CIRP Annals - Manufacturing Technology, vol. 55, no. 1, pp. 29 - 32, 2006. [Online]. Available: http://www.sciencedirect.com/science/ article/B8CXH-4P37B21-7/2/ccd599e08062dd1a48024f294c5bab7b

[14] M. Hägele, J. Neugebauer, and R. Schraft, "From robots to robot assistants," in Proc. of the 32nd ISR (International Symposium on Robotics), 2001, pp. 19-21.

[15] K. Kosuge, H. Yoshida, D. Taguchi, T. Fukuda, K. Hariki, K. Kanitani, and M. Sakai, "Robot-human collaboration for new robotic applications," in 20th International Conference on Industrial Electronics, Control and Instrumentation, vol. 2, 1994.

[16] K. Kosuge, M. Sato, and N. Kazamura, "Mobile robot helper," in Proceedings of the IEEE International Conference on Robotics and Automation, vol. 1, 2000, pp. 583-588.

[17] O. Khatib, "Mobile manipulation: The robotic assistant," Robotics and Autonomous Systems, vol. 26, no. 23, pp. 175-183, 1999, field and Service Robotics. [Online]. Available: http://www.sciencedirect.com/science/article/ B6V16-3WG3JVF-7/2/d18817e8dec02bdea88eb5d80bd9f76b

[18] E. Colgate, W. Wannasuphoprasit, and M. A. Peshkin, "Cobots: Robots for collaboration with human operators," in Proceedings of the International Mechanical Engineering Conference And Exhibition, vol. 58, 1996, pp. 433-439.

[19] J. Pires and J. Sá da Costa, "Object-oriented and distributed approach for programming robotic manufacturing cells," Robotics and Computer Integrated Manufacturing, vol. 16, no. 1, pp. 29-42, 2000.

[20] E. Helms, R. D. Schraft, and M. Hägele, "rob@work: Robot assistant in industrial environments," in Proceedings of the 11th IEEE International Workshop on Robot and Human interactive Communication, Berlin, Germany, 2002, pp. 399-404.

[21] M. Hägele, W. Schaaf, and E. Helms, "Robot assistants at manual workplaces: Effective co-operation, and safety aspects," in In Proceedings of the 33rd International Symposium on Robotics, Stockholm, Schweden, oct 2002.

[22] S. Thiemermann and O. Schulz, "team@work mensch-roboterkooperation in der montage," Automatisierungstechnische Praxis atp: Praxis der Mess-, Steuerungs-, Regelungs-, und Informationstechnik, vol. 45 , no. 11 , pp. 31-35, 2003.

[23] S. Thiemermann, "Direct man-robot-cooperation in assembly of small volume products with a scara-robot," Ph.D. dissertation, Fraunhofer IPA, 2005.

[24] A. Stopp, T. Baldauf, S. Horstmann, and S. Kristensen, "Dynamic work space surveillance for mobile robot assistants," in Proceedings of the 12th IEEE International Workshop on Robot and Human interactive Communication (ROMAN), Millbrae, California, USA, October 2003, pp. 25-30.

[25] A. Stopp, T. Baldauf, S. Hantsche, S. Horstmann, S. Kristensen, 
F. Lohnert, C. Priem, and B. Rüscher, "The manufacturing assistant: Safe, interactive teaching of operation sequences," in Proceedings of the 11th IEEE International Workshop on Robot and Human interactive Communication (ROMAN), Berlin, Germany, 2002.

[26] I. Iossifidis, C. Bruckhoff, C. Theis, C. Grote, C. Faubel, and G. Schoner, "CORA: An anthropomorphic robot assistant for human environment," in Proceedings of the 11th IEEE International Workshop on Robot and Human Interactive Communication, 2002, pp. 392-398.

[27] T. Gecks and D. Henrich, "Simero: Camera supervised workspace for service robots," in 2nd Workshop on Advances in Service Robotics (ASER), Feldafing, Germany, may 2004.

[28] M. Fischer and D. Henrich, "3d collision detection for industrial robots and unknown obstacles using multiple depth images," in Advances in Robotics Research, T. Kröger and F. M. Wahl, Eds. Springer Berlin Heidelberg, 2009, pp. 111-122. [Online]. Available: http://dx.doi.org/10.1007/978-3-642-01213-6_11

[29] O. Schrempf, U. Hanebeck, A. Schmid, and H. Wörn, "A novel approach to proactive human-robot cooperation," in IEEE International Workshop on Robot and Human Interactive Communication, 2005. ROMAN 2005., 13-15 Aug. 2005, pp. 555-560.

[30] M. Rickert, M. E. Foster, M. Giuliani, T. By, G. Panin, and A. Knoll, "Integrating language, vision and action for human robot dialog systems," in Proceedings of the 4th International Conference on Universal Access in Human-Computer Interaction, HCI International, Part II, ser. Lecture Notes in Computer Science, C. Stephanidis, Ed., vol. 4555. Beijing: Springer, July 2007, pp. 987-995. [Online]. Available: http://www.springerlink.com/content/ d038121x21629077/fulltext.pdf

[31] G. Hirzinger, A. Albu-Schaffer, M. Hahnle, I. Schaefer, and N. Sporer, "On a new generation of torque controlled light-weight robots," in Proceedings of the IEEE International Conference on Robotics and Automation, vol. 4, 2001, pp. 3356-3363.

[32] R. Burger, S. Haddadin, G. Plank, S. Parusel, and G. Hirzinger, "The driver concept for the dlr lightweight robot iii," in Proceedings of the IEEE/RSJ International Conference on Intelligent Robots and Systems (IROS), Oct. 2010, pp. 5453-5459.

[33] C. Ott, O. Eiberger, W. Friedl, B. Bäuml, U. Hillenbrand, C. Borst, A. Albu-Schäffer, B. Brunner, H. Hirschmüller, S. Kielhöfer, R. Konietschke, M. Suppa, T. Wimböck, F. Zacharias, and G. Hirzinger, "A humanoid two-arm system for dexterous manipulation," in Proceedings of the 6th IEEE-RAS International Conference on Humanoid Robots. IEEE, 2006, pp. 276-283.

[34] S. Haddadin, M. Suppa, S. Fuchs, T. Bodenmüller, A. Albu-Schäffer, and G. Hirzinger, "Towards the robotic co-worker," in Robotics Research, ser. Springer Tracts in Advanced Robotics, C. Pradalier, R. Siegwart, and G. Hirzinger, Eds. Springer Berlin / Heidelberg, 2011, vol. 70, pp. 261-282.

[35] J. T. C. Tan, Y. Zhang, F. Duan, K. Watanabe, R. Kato, and T. Arai, "Human factors studies in information support development for human-robot collaborative cellular manufacturing system," in The 18th IEEE International Symposium on Robot and Human Interactive Communication, 2009, Oct 2009, pp. 334-339.

[36] W. Erlhagen, A. Mukovskiy, E. Bicho, G. Panin, C. Kiss, A. Knoll, H. van Schie, and H. Bekkering, "Goal-directed imitation for robots: A bio-inspired approach to action understanding and skill learning," Robotics and Autonomous Systems, vol. 54, no. 5, pp. 353-360, 2006.

[37] C. Atkeson, J. Hale, F. Pollick, M. Riley, S. Kotosaka, S. Schaul, T. Shibata, G. Tevatia, A. Ude, S. Vijayakumar, E. Kawato, and M. Kawato, "Using humanoid robots to study human behavior," Intelligent Systems and their Applications, IEEE, vol. 15, no. 4, pp. 46-56, jul 2000.

[38] N. Sebanz, H. Bekkering, and G. Knoblich, "Joint action: bodies and minds moving together," Trends in Cognitive Sciences, vol. 10, no. 2, pp. 70-76, February 2006.

[39] G. Knoblich and J. S. Jordan, "Action coordination in groups and individuals: learning anticipatory control." J Exp Psychol Learn Mem Cogn, vol. 29, no. 5, pp. 1006-1016, September 2003.

[40] R. G. J. Meulenbroek, J. Bosga, M. Hulstijn, and S. Miedl, "Jointaction coordination in transferring objects," Experimental Brain Research, vol. 180, no. 2, pp. 333-343, 2007.

[41] M. Goodrich and D. Olsen, "Seven principles of efficient human robot interaction," in Proceedings on the IEEE International Conference on Systems Man and Cybernetics, vol. 4, 2003, pp. 3943-3948.

[42] S. Stork, C. Stößel, H. J. Müller, M. Wiesbeck, M. Zäh, and A. Schubö, "A neuroergonomic approach for the investigation of cognitive pro- cesses in interactive assembly environments," in Proceedings of the 16th IEEE International Symposium on Robot and Human interactive Communication (RO-MAN), Aug. 2007, pp. 750-755.

[43] M. Buss, M. Beetz, and D. Wollherr, "Cotesys - cognition for technical systems," in Proceedings of the 4th COE Workshop on Human Adaptive Mechatronics (HAM), 2007.

[44] A. Bauer, D. Wollherr, and M. Buss, "Human-robot collaboration: A survey," International Journal of Humanoid Robotics, vol. 5, no. 4, pp. $47-66,2008$.

[45] B. Grosz, "Collaborative systems," AI magazine, vol. 17, no. 2, pp. 67-86, 1996.

[46] F. Tang and L. E. Parker, "Peer-to-peer human-robot teaming through reconfigurable schemas," in AAAI Spring Symposium on "To Boldly Go Where No Human-Robot Team Has Gone Before", Stanford University, March 2006.

[47] G. Hoffman and C. Breazeal, "Effects of anticipatory action on human-robot teamwork efficiency, fluency, and perception of team," in HRI '07: Proceeding of the ACM/IEEE international conference on Human-robot interaction. New York, NY, USA: ACM, 2007, pp. $1-8$.

[48] M. Huber, A. Kupferberg, C. Lenz, A. Knoll, T. Brandt, and S. Glasauer, "Spatiotemporal movement planning and rapid adaptation for manual interaction," PLoS ONE, vol. 8, no. 5, p. e64982, 2013.

[49] M. Huber, C. Lenz, C. Wendt, B. Färber, A. Knoll, and S. Glasauer, "Predictive mechanisms increase efficiency in robot-supported assembies: An experimental evaluation," in Proceedings of the IEEE International Symposium on Robot and Human Interactive Communication, Gyengju, Korea, 2013.

[50] A. Kupferberg, M. Huber, B. Helfer, C. Lenz, A. Knoll, and S. Glasauer, "Moving just like you: Motor interference depends on similar motility of agent and observer," PLOS ONE, vol. 7, no. 6, p. 39637, 2012.

[51] C. Lenz, M. Grimm, T. Röder, and A. Knoll, "Fusing multiple kinects to survey shared human-robot-workspaces," Technische Universität München, Munich, Germany, Tech. Rep. TUM-I1214, 2012.

[52] C. Lenz, A. Sotzek, T. Röder, H. Radrich, A. Knoll, M. Huber, and S. Glasauer, "Human workflow analysis using $3 \mathrm{~d}$ occupancy grid hand tracking in a human-robot collaboration scenario," in Proceedings of the IEEE/RSJ International Conference on Intelligent Robots and Systems, San Francisco, USA, 2011. 